\title{
EFFECT OF FEEDING PALM OIL SLUDGE AS PARTIAL REPLACEMENT OF MAIZE IN GROWING-FINISHING PIGS ON THE GROWTH PERFORMANCE, NUTRIENT DIGESTIBILITY AND BLOOD PROFILES
}

\author{
TEMJENNUNGSANG*; SAMANTA, A K*; ALI, M A**; DAS, B K* and GIRIN, G K
}

\begin{abstract}
The study was conducted to investigate the effect of dietary inclusion of palm oil sludge (POS) as partial replacement of maize in growing-finishing pigs on growth performance, nutrient digestibility and blood profiles. Twenty four crossbred (Large White Yorkshire $x$ Hampshire) pigs of mixed sex (3-4 months of age) with initial body weight of $40.09 \pm 1.01 \mathrm{~kg}$ were randomly allotted to four dietary treatments in a randomised block design. Palm oil sludge was included in the diets at 0\% (Group-1, control), 10\% (Group-2), 20\% (Group-3) and 30\% (Group-4) to replace maize as energy source. The body weight gain, average daily feed intake, average daily gain and feed conversion ratio were similar across the treatment groups. The inclusion of POS in the diets had no effect on the haematological parameters, serum metabolites and lipid profile. The digestibility coefficient adverse of ether extract (EE) and crude fibre (CF) were not affected by dietary POS supplementation but dry matter (DM), organic matter (OM), CF and nitrogen free extract (NFE) revealed significant $(p<0.05)$ difference among the various treatment groups. In conclusion, POS can replace maize up to $30 \%$ in the concentrate diets without any detrimental effects on the growth performance, nutrient utilisation and blood biochemical parameters of growing-finishing pigs.
\end{abstract}

Keywords: digestibility coefficient, growing-finishing pigs, growth performance, haemato-biochemical parameters, palm oil sludge.

Received: 14 June 2019; Accepted: 20 July 2020; Published online: 7 October 2020.

\section{INTRODUCTION}

Animal husbandry plays a vital role in the economic development of Mizoram, India and pig is by far the most populous which is reared in each and every house hold. However, the inadequate supply of

\footnotetext{
Department of Animal Nutrition,

College of Veterinary Sciences and Animal Husbandry,

CAU, Selesih, Aizawl, Mizoram, India.

E-mail: temjen76@gmail.com

** Department of Veterinary Biochemistry,

College of Veterinary Sciences and Animal Husbandry, CAU, Selesih, Aizawl, Mizoram, India.

‡ Department of Livestock and Management, College of Veterinary Sciences and Animal Husbandry, CAU, Selesih, Aizawl, Mizoram, India.
}

feed, both quantitatively and qualitatively and high cost of feed ingredients are the principal limiting factors for efficient pig production. Therefore, there is a continuous quest for other cheap and indigenous sources of alternative feedstuffs. Agroindustrial by-products have become important feed components in livestock and poultry diets mainly due to the increased competition for the conventional feed ingredients, especially cereals by humans and the food industries. Hence, a byproduct of interest in palm oil production in terms of substituting energy in conventional monogastric diet is palm oil sludge (POS). Palm oil mill effluent (POME) is a general description for the discharge from palm oil extraction in the mill and is composed of various liquid, dirt, residual oil and 
suspended solids, mainly cellulosic material from the mesocarp of the fruits. POS or decanter cake is a by-product obtained after dehydration of POME. Hence, POS is a potential environmental pollutant and its utilisation as animal feed will minimise the environmental problem as well as provide energy for the animal (Webb et al., 1977). The nutrient content of POS varies considerably depending on the variety (Bamikole and Ikatua, 2009) and the method of oil extraction (Seephueak et al., 2011) but Devendra (1977) had reported the comparability of POS to maize in terms of chemical composition. Its composition is superior to that of maize, particularly with respect to protein and energy.

The use of POS as an unconventional feed in pigs has been substantiated by Vroom (1978); Hertrampt (1988); Perez (1997) and Ezekwe et al. (2011). Research on feeding of POS in pigs has so far not been carried out scientifically in India. There is a scope for utilisation of POS as a potential energy source for livestock as an alternative for maize in areas where oil palm industries are located. Therefore, this study was undertaken to evaluate the potential of POS as partial replacement of maize on the performance, nutrient utilisation and biochemical parameters of growing-finishing pigs.

\section{MATERIALS AND METHODS}

\section{Study Location}

The feeding trial was conducted at the Instructional Livestock Farm Complex (ILFC), College of Veterinary Science and Animal Husbandry, Selesih, Aizawl, Mizoram, India. Mizoram is a North Eastern state of India located at $92^{\circ} 15^{\prime} \mathrm{E}$ to $93^{\circ} 29^{\prime} \mathrm{E}$ longitude and $21^{\circ} 58^{\prime} \mathrm{N}$ to $24^{\circ} 35^{\prime} \mathrm{N}$ latitude with a total geographical area of $21081 \mathrm{~km}^{2}$. The state is rich in flora, which include tropical trees and plants. The people of the state are agrarian and oil palm cultivation is one of the major farming activities. Currently, Mizoram is producing about $6500 \mathrm{t}$ of palm oil (MOFPI, 2017).

\section{Experimental Animal and Their Management}

Twenty four crossbred (Large White Yorkshire $\mathrm{x}$ Hampshire) pigs were taken from the Instructional Livestock farm of College of Veterinary Sciences and Animal Husbandry, Selesih. Transfer of animals to the experimental shed was done and care was taken to cause minimal stress during transportation. The pigs were housed individually under intensive system of management. The adaptation period was for 14 days during which the groups to be fed with experimental feeds were given an actual amount on dry matter basis depending upon their group. All the animals were vaccinated against swine fever disease and dewormed before the commencement of the trial.

\section{Experimental Design}

Twenty four crossbred (Large White Yorkshire $x$ Hampshire) growing pigs aged 3-4 months $(40.0 \pm 91.01 \mathrm{~kg})$ were assigned to four treatments in a completely randomised design with six pigs per treatment. Feeding programme was conducted in two phases i.e. Phase I (20-50 kg) and Phase II (50$80 \mathrm{~kg}$ ) for total experimental period of 10 weeks, as per National Research Council (NRC) 1998. There were four dietary treatments; Group-1 (Control, 0\% POS), Group-2 (10\% of replacement of maize with POS), Group-3 (20\% replacement of maize with POS) and Group-4 (30\% replacement of maize with POS). The diets formulated were all isonitrogenous and isocaloric.

\section{Feed Intake}

Feeding was done individually and drinking water was made available at all times. The feeds were offered twice to the animals at $9.00 \mathrm{am}$ in the morning and at $4.00 \mathrm{pm}$ in the evening. The daily feed intake was recorded from the difference between the amount of feed offered and the residue left in the feeding throughout the day (24 hr).

\section{Body Weight Changes and Feed Conversion Efficiency}

Individual body weights of the pigs were recorded before the start of the feeding experiment and thereafter at fortnightly intervals before feeding and water provision in the morning, using an electronic scale to assess the body weight changes and growth rate. Feed efficiency during the respective as well as overall periods was calculated as the ratio between the feed intake per unit body weight gain.

\section{Haemato-biochemical Analysis}

Blood sample was collected aseptically from the anterior vena cava using $10 \mathrm{ml}$ syringe at the onset and Day 50 of the feeding trial. Haematological parameters were estimated with the help of Automated Blood Cell Counter (Model: MS4E, The Netherland) by following the standard procedures as per the manufacturers' protocol. Blood biochemical parameters were estimated with the help of UV Visible spectrophotometer (Serial No. A114518; Shimadzu Corp.) using commercially available diagnostic kits (Coral diagnostics). 


\section{Digestibility Trial}

For the digestibility trial, four animals from each group were randomly selected. Three days adaptation period was given to animals prior to sampling and collection. During the digestibility trial, individual feed intake, residue left and faeces voided by the animals were recorded individually. Wholesome drinking water was made available to all animals ad libitum. The digestibility trial was conducted for four days after the end of the feeding trial and representative faecal sample and feed was collected and processed for chemical analysis.

\section{Chemical Analysis}

Proximate principles and fibre fractions. POS, feed and faeces were analysed (AOAC,1995) for dry matter (DM; method 934.01), crude protein (CP; method 968.06; Kelplus, Pelican Equipments, Chennai, India), crude fibre (CF; Fibreplus, Pelican Equipments, Chennai, India) and ether extract (EE; method 920.39; Socsplus, Pelican Equipments, Chennai, India). The fibre fractions were determined as per the method described by Goering and Van Soest (1970). Calcium was estimated as per Talapatra et al. (1940), whereas phosphorus was estimated as per Association of Official Analytical Chemists (AOAC) (1995). All the samples for the analysis were weighed using an electronic balance (Model No. ABS 220-4, Kern and Sohn GmbH). The digestible energy was calculated using the prediction equation described by Noblet and Perez (1993).

\section{Fatty Acid Analysis}

Fatty acids content of POS was determined following the method of O'Fallon et al. (2007) with slight modification (Mandal et al., 2014). In brief, 0.5 g sample was placed into a screw-cap pyrex culture tube to which $0.5 \mathrm{ml}$ of the $\mathrm{C} 13: 0$ internal standard ( $0.5 \mathrm{mg}$ of $\mathrm{C} 13: 0 \mathrm{ml}$ in methanol), $0.35 \mathrm{ml}$ of $10 \mathrm{~N} \mathrm{KOH}$ in water and $2.65 \mathrm{ml}$ of methanol was added. The tube was incubated at $55^{\circ} \mathrm{C}$ for $1.5 \mathrm{hr}$ with vigorous hand-shaking for $5 \mathrm{~s}$ every $20 \mathrm{~min}$ to properly permeate, dissolve and hydrolyse the sample. After cooling below room temperature in a cold tap water bath, $0.29 \mathrm{ml}$ of $24 \mathrm{~N} \mathrm{H}_{2} \mathrm{SO}_{4}$ in water was added. The tube was mixed thoroughly and incubated again at $55^{\circ} \mathrm{C}$ for $1.5 \mathrm{hr}$ with hand-shaking for $5 \mathrm{~s}$ every 20 min. After fatty acid methyl ester (FAME) synthesis, the tube was cooled in a cold tap water bath, 1.5 $\mathrm{ml}$ of hexane was added, and the tube was mixed for $5 \mathrm{~min}$ on a multi-tube vortex. The tube was centrifuged for $5 \mathrm{~min}$ in a table top centrifuge at 2500 rpm and the hexane layer containing FAME was placed into a vial. The vial was capped and placed at $-20^{\circ} \mathrm{C}$ until analysis. The prepared FAME was determined by capillary gas chromatography (GC) on a HP-88, $100 \mathrm{~m} \times 0.25 \mathrm{~mm} \times 0.20 \mu \mathrm{m}$ capillary column (Agilent Technologies Inc., Santra Clara, California, USA) with a flame ionisation detector. Identification and quantification of individual FA were performed using a purified standard (Supelco 37 component FAME mixture, SigmaAldrich Chemical Pvt. Ltd., Kolkata, India) and an internal standard (tridecanoic acid; Sigma-Aldrich chemical Pvt. Ltd. Kolkata, India). The fatty acid concentration of POS was expressed as g per $100 \mathrm{~g}$ of the sum of identified peaks measured in each sample.

\section{Statistical Analysis}

The data were analysed using general linear model procedure of statistical analysis system (SAS, 2003). The linear and quadratic effects of dietary increasing proportion of inclusion of POS against the control group on performance, haemato-biochemical and nutrient digestibility was determined by orthogonal polynomial contrasts. One-way analysis of variance (ANOVA) has been applied for comparison among treatments and paired t-test was performed for comparison between periods (Day 0 and Day 50) with in group. Tukey's test was used to compare the means of the treatment with probability of $5 \%$ $(\mathrm{p}<0.05)$.

The statistical model used for one-way ANOVA was:

$Y_{i j}=\mu+\tau_{i j}+\varepsilon_{i j}$

where,

$Y_{i j}=$ response from the $j^{\text {th }}$ unit receiving $i^{\text {th }}$ treatment

$\mu=$ general mean effect

$\tau_{\mathrm{ij}}=$ the effect due to $\mathrm{i}^{\text {th }}$ treatment

$\varepsilon_{\mathrm{ij}}=$ the random error.

\section{RESULTS}

\section{Chemical Composition of POS and Experimental Diets}

The result on the chemical composition, fibre fraction and fatty acid composition of POS is shown in Table 1. The proximate principles $\left(\mathrm{g} \mathrm{kg}^{-1} \mathrm{DM}\right)$ i.e. moisture, crude protein $(\mathrm{CP})$, ether extract (EE), crude fibre (CF), nitrogen free extract (NFE) and total ash (TA) in POS was 722.91, 138.70, 101.97, 214.75, 315.03 and 229.55, respectively. The POS contained on an average $72.80\left(\mathrm{~g} \mathrm{~kg}^{-1} \mathrm{DM}\right)$ acid insoluble ash (AIA). The fibre fractions for neutral detergent fibre (NDF) and acid detergent fibre (ADF) was 568.00 and $547.00\left(\mathrm{~g} \mathrm{~kg}^{-1} \mathrm{DM}\right)$, respectively. 
The individual fatty acid composition of POS for C10:0, C12:0, C14:0, C14:1, C15:0, C16:0, C16:1, C17:0, C18:0, C18:1 cis, C18:2 cis, C18:3n6, C18:3n3, C20:1 and C24:0 is presented in Table 1. The sum of saturated fatty acid (SFA), monounsaturated fatty acid (MUFA), polyunsaturated fatty acid (PUFA) and ratio of PUFA to SFA in POS was 62.84, $25.78,10.75$ (g per $100 \mathrm{~g}$ fatty acid) and 0.17, respectively.

The chemical composition of the experimental diets fed to the growing-finishing pigs during Phase I and Phase II stages were analysed and is presented in Table 2. The chemical compositions of the experimental diets were similar in the two phases and between the groups i.e. the diets were iso-nitrogenous and iso-caloric.

TABLE 1. PROXIMATE COMPOSITION, FIBRE FRACTION ( $\mathrm{g} \mathrm{kg}^{-1} \mathrm{DM}$ ) AND FATTY ACID COMPOSITION (g per $100 \mathrm{~g}$ fatty acid) OF PALM OIL SLUDGE (POS)

\begin{tabular}{|c|c|}
\hline Attributes & POS \\
\hline \multicolumn{2}{|l|}{ Proximate composition ( $\left.\mathrm{g} \mathrm{kg}^{-1} \mathrm{DM}\right)$} \\
\hline Moisture & 722.91 \\
\hline Crude protein & 138.70 \\
\hline Ether extract & 101.97 \\
\hline Crude fibre & 214.75 \\
\hline Nitrogen free extract & 315.03 \\
\hline Total ash & 229.55 \\
\hline Acid insoluble ash & 72.80 \\
\hline Digestible energy $\left(\mathrm{Kcal} \mathrm{kg}^{-1}\right)$ & 3535.21 \\
\hline \multicolumn{2}{|l|}{ Fibre fractions ( $\left.\mathrm{g} \mathrm{kg}^{-1} \mathrm{DM}\right)$} \\
\hline Neutral detergent fibre & 568.00 \\
\hline Acid detergent fibre & 547.00 \\
\hline \multicolumn{2}{|c|}{ Fatty acid composition (g per $100 \mathrm{~g}$ fatty acid) } \\
\hline Capric acid (C10:0) & 0.03 \\
\hline Lauric acid (C12:0) & 0.08 \\
\hline Myristic acid (C14:0) & 0.95 \\
\hline Myristoleic acid (C14:1) & 0.35 \\
\hline Pentadecylic acid (C15:0) & 0.09 \\
\hline Palmitic acid (C16:0) & 53.38 \\
\hline Palmitoleic acid (C16:1) & 0.13 \\
\hline Margaric acid (C17:0) & 0.12 \\
\hline Stearic acid (C18:0) & 8.06 \\
\hline Oleic acid (C18:1 cis) & 25.23 \\
\hline Linoleic acid (C18:2 cis) & 10.13 \\
\hline Gamma linolenic acid (C18:3n6) & 0.40 \\
\hline Alpha linolenic acid (C18:3n3) & 0.22 \\
\hline Gondoic acid (C20:1) & 0.07 \\
\hline Lignoceric acid (C24:0) & 0.13 \\
\hline Total & 99.37 \\
\hline$\Sigma$ SFA & 62.84 \\
\hline$\Sigma$ MUFA & 25.78 \\
\hline$\Sigma$ PUFA & 10.75 \\
\hline PUFA:SFA & 0.17 \\
\hline
\end{tabular}

Note: POS - palm oil sludge; SFA - saturated fatty acid; MUFA monounsaturated fatty acid; PUFA - polyunsaturated fatty acid.

\section{Feed and Nutrient Intake}

As shown in Table 3, the average daily DM intake was comparable throughout the experimental period.

Body weight changes and feed conversion ratio. The results pertaining to the performance of pigs fed increasing levels of POS are shown in Table 3. The average weight gain, average daily gain (ADG) and feed conversion ratio (FCR) were similar in all the groups in both Phase-I and Phase-II. Overall average for the above parameters was also similar in all the groups. Among the treatments, the ADG showed a marginal increase and a better FCR in Group-2 and Group-4 during the Phase-I feeding. In the Phase II, lesser ADG and higher FCR was observed in the POS fed animals when compared to the control. Group-3 recorded the lowest ADG and highest FCR in the overall of the experimental period. However, both ADG and FCR remained comparable throughout the experiment, irrespective of treatments.

\section{Haemato-biochemical Profiles}

Increasing levels of POS supplementation in the concentrate mixtures (Table 4) had no effect on the concentration of haematological parameters like haemoglobin $(\mathrm{Hb})$, white blood cells (WBC), red blood cells (RBC), packed cell volume (PCV), mean corpuscular volume (MCV) and lymphocytes. However, the mean corpuscular haemoglobin $(\mathrm{MCH})$ levels revealed a quadratic $(\mathrm{p}<0.05)$ response and a linear $(\mathrm{p}<0.05)$ effect in the monocyte levels among the treatment groups. A linear and quadratic increase $(p<0.01)$ in the level of mean corpuscular haemoglobin concentration (MCHC) was observed in Group-4 on the Day 50 of the experiment. Significant difference $(\mathrm{p}<0.05)$ was observed in the level of $\mathrm{Hb}, \mathrm{RBC}, \mathrm{MCV}$, mean corpuscular haemoglobin, lymphocytes and monocytes between two periods i.e. Day 0 and Day 50 in 30\% POS supplemented group (Group-4). Level of MCV, MCH and lymphocytes were also significantly different $(\mathrm{p}<0.05)$ between two periods within control and 10\% POS supplemented group.

The results pertaining to serum metabolites are presented in Table 5. The concentration of serum glutamic pyruvic transaminase (SGPT), serum glutamic oxaloacetic transaminase (SGOT), alkaline phosphatase (ALP), creatinine, urea, glucose, total protein, albumin, globulin, calcium (Ca) and phosphorus (P) in the serum was similar in the entire group throughout the experimental period. Similarly, the concentration of triglyceride, cholesterol, high density lipoprotein (HDL) and low density lipoprotein (LDL) also showed no variation among the various treatment groups. There were no significant difference in the concentration of blood 
TABLE 2. INGREDIENT AND CHEMICAL COMPOSITION (\%) OF THE EXPERIMENTAL DIETS

\begin{tabular}{|c|c|c|c|c|c|c|c|c|}
\hline \multirow[b]{2}{*}{ Attributes } & \multicolumn{4}{|c|}{ Phase I (20-50 kg) } & \multicolumn{4}{|c|}{ Phase II (50-80 kg) } \\
\hline & $\begin{array}{c}\text { Group-1 } \\
(0 \% \text { POS })\end{array}$ & $\begin{array}{c}\text { Group-2 } \\
\text { (10\%POS) }\end{array}$ & $\begin{array}{c}\text { Group-3 } \\
(20 \% \text { POS })\end{array}$ & $\begin{array}{c}\text { Group-4 } \\
\text { (30\%POS) }\end{array}$ & $\begin{array}{l}\text { Group-1 } \\
(0 \% \text { POS) }\end{array}$ & $\begin{array}{c}\text { Group-2 } \\
\text { (10\%POS) }\end{array}$ & $\begin{array}{c}\text { Group-3 } \\
\text { (20\%POS) }\end{array}$ & $\begin{array}{c}\text { Group-4 } \\
\text { (30\% POS) }\end{array}$ \\
\hline Maize & 60 & 54 & 48 & 42 & 68 & 61.2 & 54.4 & 47.6 \\
\hline Palm oil sludge & - & 6 & 12 & 18 & - & 6.8 & 13.6 & 20.4 \\
\hline Soyabean meal & 19.44 & 19.44 & 19.44 & 19.45 & 13.97 & 13.97 & 14.15 & 14.14 \\
\hline Wheat bran & 12.5 & 11 & 10.5 & 9.8 & 12 & 11 & 9.2 & 9.1 \\
\hline Palm oil & - & 1.5 & 2 & 2.7 & - & 1 & 2 & 2.7 \\
\hline Fish meal & 6 & 6 & 6 & 6 & 4 & 4 & 4 & 4 \\
\hline Lysine & 0.06 & 0.06 & 0.06 & 0.05 & 0.03 & 0.03 & 0.05 & 0.06 \\
\hline Mineral mixture & 1.5 & 1.5 & 1.5 & 1.5 & 1.5 & 1.5 & 1.5 & 1.5 \\
\hline Salt & 0.5 & 0.5 & 0.5 & 0.5 & 0.5 & 0.5 & 0.5 & 0.5 \\
\hline Total & 100 & 100 & 100 & 100 & 100 & 100 & 100 & 100 \\
\hline \multicolumn{9}{|l|}{ Calculated } \\
\hline Crude protein & 18.12 & 17.99 & 17.99 & 17.97 & 15.57 & 15.50 & 15.51 & 15.48 \\
\hline Digestible energy $\left(\mathrm{Kcal} \mathrm{kg}^{-1}\right)$ & 3352.06 & 3414.03 & 3423.20 & 3443.30 & 3368.31 & 3401.58 & 3436.58 & 3453.65 \\
\hline \multicolumn{9}{|l|}{ Estimated } \\
\hline Crude protein & 17.89 & 17.67 & 18.16 & 18.32 & 15.86 & 15.66 & 15.53 & 15.61 \\
\hline Ether extract & 3.71 & 4.67 & 4.98 & 5.67 & 4.60 & 5.21 & 7.70 & 9.72 \\
\hline Crude fibre & 3.12 & 3.56 & 4.01 & 4.67 & 3.32 & 5.24 & 5.92 & 6.34 \\
\hline Total ash & 6.23 & 6.78 & 7.34 & 8.17 & 7.41 & 8.45 & 8.97 & 10.05 \\
\hline Acid insoluble ash & 1.03 & 1.78 & 2.17 & 2.35 & 1.12 & 1.67 & 2.29 & 2.57 \\
\hline Digestible energy $\left(\mathrm{Kcal} \mathrm{kg}^{-1}\right)$ & 3445.94 & 3413.80 & 3405.52 & 3384.48 & 3380.89 & 3420.73 & 3346.41 & 3255.26 \\
\hline
\end{tabular}

TABLE 3. GROWTH PERFORMANCE OF GROWING-FINISHING PIGS FED PALM OIL SLUDGE (POS) BASED DIETS

\begin{tabular}{|c|c|c|c|c|c|c|c|}
\hline \multirow{2}{*}{ Period } & \multirow{2}{*}{$\begin{array}{l}\text { Group-1 } \\
\text { (0\% POS) }\end{array}$} & \multirow{2}{*}{$\begin{array}{c}\text { Group-2 } \\
\text { (10\% POS) }\end{array}$} & \multirow{2}{*}{$\begin{array}{c}\text { Group-3 } \\
(20 \% \text { POS) }\end{array}$} & \multirow{2}{*}{$\begin{array}{c}\text { Group-4 } \\
\text { (30\% POS) }\end{array}$} & \multirow{2}{*}{ SEM } & \multicolumn{2}{|c|}{ Contrast, p-value } \\
\hline & & & & & & Linear & Quadratic \\
\hline \multicolumn{8}{|l|}{ Phase I } \\
\hline Initial weight (kg) & 40.04 & 40.13 & 41.44 & 40.16 & 1.000 & 0.860 & 0.754 \\
\hline Final weight $(\mathrm{kg})$ & 48.76 & 49.72 & 49.79 & 49.72 & 0.910 & 0.734 & 0.797 \\
\hline Ave. wt. gain (kg) & 8.72 & 9.59 & 8.35 & 9.56 & 0.363 & 0.701 & 0.819 \\
\hline $\mathrm{DMI}(\mathrm{g} / \mathrm{h} / \mathrm{d})$ & 2160.60 & 2186.19 & 1995.71 & 2216.07 & 52.06 & 0.959 & 0.324 \\
\hline $\operatorname{ADG}(\mathrm{g} / \mathrm{h} / \mathrm{d})$ & 623.09 & 684.76 & 596.57 & 682.86 & 25.90 & 0.701 & 0.819 \\
\hline FCR & 3.47 & 3.20 & 4.07 & 3.29 & 0.227 & 0.886 & 0.592 \\
\hline \multicolumn{8}{|l|}{ Phase II } \\
\hline Initial weight (kg) & 48.76 & 49.72 & 49.79 & 49.72 & 0.910 & 0.734 & 0.797 \\
\hline Final weight $(\mathrm{kg})$ & 82.80 & 82.01 & 81.68 & 82.98 & 0.880 & 0.981 & 0.586 \\
\hline Ave. wt. gain (kg) & 34.04 & 32.30 & 32.89 & 33.26 & 0.710 & 0.667 & 0.308 \\
\hline $\mathrm{DMI}(\mathrm{g} / \mathrm{h} / \mathrm{d})$ & 2612.00 & 2627.00 & 2564.20 & 2659.70 & 20.73 & 0.672 & 0.355 \\
\hline $\operatorname{ADG}(\mathrm{g} / \mathrm{h} / \mathrm{d})$ & 607.92 & 576.79 & 569.50 & 593.93 & 12.68 & 0.677 & 0.308 \\
\hline FCR & 4.32 & 4.58 & 4.56 & 4.56 & 0.101 & 0.464 & 0.540 \\
\hline \multicolumn{8}{|l|}{ Overall average } \\
\hline Initial weight (kg) & 40.04 & 40.13 & 41.44 & 40.16 & 1.000 & 0.860 & 0.754 \\
\hline Final weight $(\mathrm{kg})$ & 82.80 & 82.01 & 81.68 & 82.98 & 0.880 & 0.981 & 0.586 \\
\hline Ave. wt. gain (kg) & 42.76 & 41.89 & 40.24 & 42.82 & 0.916 & 0.862 & 0.380 \\
\hline $\mathrm{DMI}(\mathrm{g} / \mathrm{h} / \mathrm{d})$ & 2521.70 & 2539.50 & 2450.50 & 2571.00 & 24.99 & 0.793 & 0.320 \\
\hline $\operatorname{ADG}(\mathrm{g} / \mathrm{h} / \mathrm{d})$ & 610.95 & 598.38 & 574.92 & 611.72 & 13.08 & 0.862 & 0.380 \\
\hline FCR & 4.21 & 4.45 & 4.62 & 4.46 & 0.139 & 0.471 & 0.522 \\
\hline
\end{tabular}

Note: DMI - dry matter intake; ADG - average daily gain; FCR - feed conversion ratio; Ave. wt. - average weight; SEM - standard error of mean. 
biochemical parameters between the two periods i.e. Day 0 and Day 50 within groups except concentration of ALP, cholesterol, LDL and Ca and concentration of LDL and $\mathrm{Ca}$, which were significantly different $(p<0.05)$ between the period within control group and $30 \%$ POS supplemented group, respectively.

\section{Apparent Digestibility of DM and Other Nutrients}

The effect of feeding graded levels of POS in the concentrate as partial replacement of maize on nutrient digestibility of the growing-finishing pigs is presented in Table 6. Feeding of increasing levels of POS so as to replace $0 \%, 10 \%, 20 \%$ and $30 \%$ of maize led to a linear decrease $(p<0.01)$ in the digestibility coefficient of DM, organic matter,
$\mathrm{CP}$ and nitrogen free extract. The digestibility coefficient of all the above components was higher in Group-1 and lowest in Group-4. However, the digestibility coefficient of ether extract and crude fibre was similar among the dietary treatments.

\section{Economics}

Feed economics of POS based concentrate fed to growing-finishing pigs is shown in Table 6. The cost of feed per weight gain in all the groups was similar. However, the average total feed cost had linearly decreased $(\mathrm{p}<0.01)$ in the treatment groups as the level of POS was increased in the concentrate diets.

TABLE 4. EFFECT OF PALM OIL SLUDGE (POS) ON HAEMATOLOGICAL PARAMETERS OF GROWING-FINISHING PIGS

\begin{tabular}{|c|c|c|c|c|c|c|c|}
\hline \multirow{2}{*}{ Attributes } & \multirow{2}{*}{$\begin{array}{l}\text { Group-1 } \\
(0 \% \text { POS) }\end{array}$} & \multirow{2}{*}{$\begin{array}{c}\text { Group-2 } \\
(10 \% \text { POS) }\end{array}$} & \multirow{2}{*}{$\begin{array}{c}\text { Group-3 } \\
(20 \% \text { POS) }\end{array}$} & \multirow{2}{*}{$\begin{array}{c}\text { Group-4 } \\
(30 \% \text { POS) }\end{array}$} & \multirow{2}{*}{ SEM } & \multicolumn{2}{|c|}{ Contrast, p-value } \\
\hline & & & & & & Linear & Quadratic \\
\hline \multicolumn{8}{|l|}{ Haemoglobin (g/dl) } \\
\hline Day 0 & 12.43 & 12.88 & 11.93 & 11.20 & 0.392 & 0.221 & 0.477 \\
\hline Day 50 & 12.45 & 13.03 & 11.39 & 13.67 & 0.320 & 0.410 & 0.134 \\
\hline p-value & 0.986 & 0.877 & 0.504 & 0.014 & & & \\
\hline \multicolumn{8}{|c|}{ White blood corpuscle $\left(\mathrm{m} / \mathrm{mm}^{3}\right)$} \\
\hline Day 0 & 15.15 & 18.94 & 15.39 & 16.77 & 0.600 & 0.777 & 0.261 \\
\hline Day 50 & 16.77 & 14.44 & 16.78 & 17.75 & 0.711 & 0.422 & 0.270 \\
\hline p-value & 0.432 & 0.116 & 0.521 & 0.686 & & & \\
\hline \multicolumn{8}{|c|}{ Red blood cells $\left(\mathrm{m} / \mathrm{mm}^{3}\right)$} \\
\hline Day 0 & 5.90 & 6.98 & 6.83 & 5.87 & 0.216 & 0.886 & 0.020 \\
\hline Day 50 & 6.35 & 7.03 & 5.51 & 7.24 & 0.280 & 0.610 & 0.302 \\
\hline $\mathrm{p}$-value & 0.589 & 0.922 & 0.202 & 0.041 & & & \\
\hline \multicolumn{8}{|l|}{ Packed cell volume (\%) } \\
\hline Day 0 & 36.20 & 41.85 & 39.28 & 36.13 & 1.174 & 0.784 & 0.073 \\
\hline Day 50 & 45.03 & 45.57 & 37.47 & 44.37 & 1.268 & 0.307 & 0.158 \\
\hline p-value & 0.135 & 0.324 & 0.647 & 0.108 & & & \\
\hline \multicolumn{8}{|c|}{ Mean corpuscular volume (fl) } \\
\hline Day 0 & 60.45 & 60.03 & 58.68 & 58.48 & 0.467 & 0.103 & 0.905 \\
\hline Day 50 & 60.55 & 61.70 & 60.40 & 61.30 & 0.337 & 0.763 & 0.859 \\
\hline p-value & 0.025 & 0.025 & 0.130 & 0.004 & & & \\
\hline \multicolumn{8}{|c|}{ Mean corpuscular haemoglobin (pg) } \\
\hline Day 0 & 17.80 & 18.00 & 17.68 & 17.68 & 0.095 & 0.446 & 0.624 \\
\hline Day 50 & $18.88^{\mathrm{a}}$ & $18.53^{\mathrm{ab}}$ & $17.60^{\mathrm{b}}$ & $18.83^{\mathrm{a}}$ & 0.192 & 0.647 & 0.008 \\
\hline p-value & 0.109 & 0.710 & 0.794 & 0.006 & & & \\
\hline \multicolumn{8}{|c|}{ Mean corpuscular haemoglobin concentration (g/dl) } \\
\hline Day 0 & 30.87 & 30.70 & 30.28 & 30.33 & 0.188 & 0.264 & 0.783 \\
\hline Day 50 & $29.75^{\mathrm{a}}$ & $29.57^{\mathrm{a}}$ & $29.47^{\mathrm{a}}$ & $30.80^{\mathrm{b}}$ & 0.165 & 0.006 & 0.003 \\
\hline p-value & 0.030 & 0.028 & 0.167 & 0.273 & & & \\
\hline \multicolumn{8}{|l|}{ Lymphocytes (\%) } \\
\hline Day 0 & 45.90 & 44.70 & 44.15 & 45.35 & 0.740 & 0.765 & 0.471 \\
\hline Day 50 & 52.68 & 51.00 & 50.20 & 51.77 & 0.510 & 0.451 & 0.135 \\
\hline $\mathrm{p}$-value & 0.018 & 0.033 & 0.064 & 0.006 & & & \\
\hline \multicolumn{8}{|l|}{ Monocytes (\%) } \\
\hline Day 0 & 5.65 & 5.38 & 6.30 & 6.18 & 0.252 & 0.304 & 0.888 \\
\hline Day 50 & $6.18^{\mathrm{a}}$ & $8.07^{\mathrm{b}}$ & $7.67^{\mathrm{b}}$ & $8.20^{\mathrm{b}}$ & 0.261 & 0.004 & 0.079 \\
\hline $\mathrm{p}$-value & 0.345 & 0.010 & 0.217 & 0.001 & & & \\
\hline
\end{tabular}

Note: ${ }^{a, b}$ means with different superscripts in the same row differ significantly $(\mathrm{p}<0.01)$; SEM - standard error of mean. 
TABLE 5. EFFECT OF PALM OIL SLUDGE (POS) ON BLOOD BIOCHEMICAL PARAMETERS OF GROWING-FINISHING PIGS

\begin{tabular}{|c|c|c|c|c|c|c|c|}
\hline \multirow{2}{*}{ Attributes } & \multirow{2}{*}{$\begin{array}{l}\text { Group-1 } \\
(0 \% \text { POS })\end{array}$} & \multirow{2}{*}{$\begin{array}{c}\text { Group-2 } \\
(\mathbf{1 0} \% \text { POS) }\end{array}$} & \multirow{2}{*}{$\begin{array}{c}\text { Group-3 } \\
\text { (20\% POS) }\end{array}$} & \multirow{2}{*}{$\begin{array}{c}\text { Group-4 } \\
\text { (30\% POS) }\end{array}$} & \multirow{2}{*}{ SEM } & \multicolumn{2}{|c|}{ Contrast, p-value } \\
\hline & & & & & & Linear & Quadratic \\
\hline \multicolumn{8}{|l|}{ SGPT (U/1) } \\
\hline Day 0 & 45.82 & 42.42 & 40.64 & 41.72 & 0.962 & 0.112 & 0.246 \\
\hline Day 50 & 47.47 & 41.35 & 44.00 & 44.76 & 1.374 & 0.671 & 0.244 \\
\hline p-value & 0.649 & 0.741 & 0.361 & 0.375 & & & \\
\hline \multicolumn{8}{|l|}{ SGOT (U/l) } \\
\hline Day 0 & 46.37 & 52.34 & 54.03 & 52.87 & 2.177 & 0.319 & 0.449 \\
\hline Day 50 & 50.03 & 53.88 & 56.62 & 56.54 & 1.733 & 0.186 & 0.512 \\
\hline $\mathrm{p}$-value & 0.599 & 0.703 & 0.465 & 0.672 & & & \\
\hline \multicolumn{8}{|l|}{$\operatorname{ALP}(\mathrm{U} / 1)$} \\
\hline Day 0 & 255.87 & 254.53 & 255.87 & 294.94 & 15.523 & 0.441 & 0.555 \\
\hline Day 50 & 195.29 & 181.65 & 143.15 & 203.85 & 11.589 & 0.899 & 0.119 \\
\hline p-value & 0.018 & 0.274 & 0.079 & 0.120 & & & \\
\hline \multicolumn{8}{|l|}{ Creatinine (mg/dl) } \\
\hline Day 0 & 1.78 & 1.56 & 1.72 & 1.71 & 0.079 & 0.937 & 0.549 \\
\hline Day 50 & 1.60 & 1.47 & 1.50 & 1.50 & 0.045 & 0.533 & 0.506 \\
\hline p-value & 0.487 & 0.429 & 0.441 & 0.330 & & & \\
\hline \multicolumn{8}{|l|}{ Urea (mg/dl) } \\
\hline Day 0 & 14.51 & 12.14 & 11.77 & 12.21 & 0.548 & 0.150 & 0.209 \\
\hline Day 50 & 14.78 & 13.78 & 15.43 & 12.30 & 0.633 & 0.316 & 0.407 \\
\hline p-value & 0.904 & 0.486 & 0.077 & 0.960 & & & \\
\hline \multicolumn{8}{|l|}{ Glucose (mg/dl) } \\
\hline Day 0 & 111.83 & 103.93 & 125.78 & 128.86 & 5.957 & 0.198 & 0.654 \\
\hline Day 50 & 106.89 & 110.44 & 90.43 & 103.83 & 4.259 & 0.455 & 0.571 \\
\hline $\mathrm{p}$-value & 0.833 & 0.520 & 0.141 & 0.094 & & & \\
\hline \multicolumn{8}{|l|}{ Total protein $(\mathrm{g} / \mathrm{dl})$} \\
\hline Day 0 & 7.04 & 7.57 & 7.32 & 7.81 & 0.143 & 0.118 & 0.950 \\
\hline Day 50 & 7.56 & 7.50 & 7.50 & 7.39 & 0.101 & 0.617 & 0.921 \\
\hline p-value & 0.148 & 0.778 & 0.535 & 0.582 & & & \\
\hline \multicolumn{8}{|l|}{ Albumin (g/dl) } \\
\hline Day 0 & 3.09 & 3.23 & 2.98 & 3.33 & 0.110 & 0.654 & 0.658 \\
\hline Day 50 & 3.50 & 3.15 & 3.18 & 3.19 & 0.102 & 0.366 & 0.413 \\
\hline $\mathrm{p}$-value & 0.104 & 0.882 & 0.712 & 0.351 & & & \\
\hline \multicolumn{8}{|l|}{ Globulin (g/dl) } \\
\hline Day 0 & 3.96 & 4.34 & 4.34 & 4.49 & 0.171 & 0.350 & 0.745 \\
\hline Day 50 & 4.07 & 4.34 & 4.33 & 4.20 & 0.158 & 0.803 & 0.574 \\
\hline $\mathrm{p}$-value & 0.530 & 1.00 & 0.983 & 0.718 & & & \\
\hline \multicolumn{8}{|c|}{ Triglycerides (mg/dl) } \\
\hline Day 0 & 28.68 & 32.91 & 32.73 & 35.00 & 1.136 & 0.077 & 0.659 \\
\hline Day 50 & 36.67 & 37.22 & 36.55 & 36.31 & 0.620 & 0.781 & 0.780 \\
\hline p-value & 0.163 & 0.030 & 0.152 & 0.737 & & & \\
\hline \multicolumn{8}{|l|}{ Cholesterol (mg/dl) } \\
\hline Day 0 & 81.68 & 94.48 & 87.41 & 96.68 & 2.684 & 0.111 & 0.726 \\
\hline Day 50 & 98.68 & 100.63 & 97.34 & 97.01 & 1.298 & 0.515 & 0.686 \\
\hline p-value & 0.023 & 0.248 & 0.374 & 0.964 & & & \\
\hline \multicolumn{8}{|l|}{ HDL (mg/dl) } \\
\hline Day 0 & 33.25 & 37.79 & 31.82 & 36.31 & 1.003 & 0.695 & 0.991 \\
\hline Day 50 & 38.13 & 42.18 & 32.94 & 33.23 & 1.703 & 0.111 & 0.557 \\
\hline $\mathrm{p}$-value & 0.126 & 0.542 & 0.716 & 0.403 & & & \\
\hline
\end{tabular}


TABLE 5. EFFECT OF PALM OIL SLUDGE (POS) ON BLOOD BIOCHEMICAL PARAMETERS OF GROWING-FINISHING PIGS (continued)

\begin{tabular}{|c|c|c|c|c|c|c|c|}
\hline \multirow{2}{*}{ Attributes } & \multirow{2}{*}{$\begin{array}{c}\text { Group-1 } \\
(0 \% \text { POS })\end{array}$} & \multirow{2}{*}{$\begin{array}{c}\text { Group-2 } \\
\text { (10\% POS) }\end{array}$} & \multirow{2}{*}{$\begin{array}{c}\text { Group-3 } \\
(20 \% \text { POS })\end{array}$} & \multirow{2}{*}{$\begin{array}{c}\text { Group-4 } \\
(30 \% \text { POS) }\end{array}$} & \multirow{2}{*}{ SEM } & \multicolumn{2}{|c|}{ Contrast, p-value } \\
\hline & & & & & & Linear & Quadratic \\
\hline \multicolumn{8}{|l|}{ LDL (mg/dl) } \\
\hline Day 0 & 36.17 & 39.69 & 38.59 & 38.37 & 1.417 & 0.698 & 0.553 \\
\hline Day 50 & 43.55 & 41.45 & 47.41 & 46.78 & 1.116 & 0.116 & 0.728 \\
\hline p-value & 0.026 & 0.473 & 0.303 & 0.023 & & & \\
\hline \multicolumn{8}{|l|}{ Calcium (mg/dl) } \\
\hline Day 0 & 6.56 & 8.65 & 7.05 & 6.86 & 0.449 & 0.061 & 1.123 \\
\hline Day 50 & 10.50 & 8.52 & 10.44 & 10.15 & 0.412 & 0.809 & 0.310 \\
\hline $\mathrm{p}$-value & 0.019 & 0.931 & 0.069 & 0.015 & & & \\
\hline \multicolumn{8}{|l|}{ Phosphorus (mg/dl) } \\
\hline Day 0 & 6.56 & 8.80 & 7.05 & 7.03 & 0.430 & 0.933 & 0.196 \\
\hline Day 50 & 8.44 & 7.94 & 8.02 & 7.54 & 0.357 & 0.349 & 0.987 \\
\hline $\mathrm{p}$-value & 0.300 & 0.421 & 0.512 & 0.525 & & & \\
\hline
\end{tabular}

Note: SGPT - serum glutamic, pyruvic transaminase; SGOT - serum glutamic oxaloacetic transaminase; ALP - alkaline phosphatase; HDL - high density lipoprotein; LDL - low density lipoprotein; SEM - standard error of mean.

TABLE 6. DIGESTIBILITY COEFFICIENT OF NUTRIENTS AND ECONOMICS OF FEEDING PALM OIL SLUDGE (POS) BASED CONCENTRATE DIETS TO GROWING-FINISHING PIGS

\begin{tabular}{|c|c|c|c|c|c|c|c|}
\hline \multirow{2}{*}{ Attributes } & \multirow{2}{*}{$\begin{array}{l}\text { Group-1 } \\
\text { (0\% POS) }\end{array}$} & \multirow{2}{*}{$\begin{array}{c}\text { Group-2 } \\
(10 \% \text { POS) }\end{array}$} & \multirow{2}{*}{$\begin{array}{c}\text { Group-3 } \\
(20 \% \text { POS })\end{array}$} & \multirow{2}{*}{$\begin{array}{c}\text { Group-4 } \\
(30 \% \text { POS) }\end{array}$} & \multirow{2}{*}{ SEM } & \multicolumn{2}{|c|}{ Contrast, p-value } \\
\hline & & & & & & Linear & Quadratic \\
\hline \multicolumn{8}{|l|}{ Digestibility coefficients } \\
\hline $\mathrm{DM}$ & $84.13^{\mathrm{b}}$ & $79.85^{\mathrm{ab}}$ & $77.27^{b}$ & $71.61^{\mathrm{a}}$ & 1.397 & $<0.01^{* *}$ & 0.695 \\
\hline $\mathrm{OM}$ & $85.82^{b}$ & $82.28^{\mathrm{b}}$ & $79.99^{\mathrm{ab}}$ & $75.74^{\mathrm{a}}$ & 1.153 & $<0.01^{* *}$ & 0.815 \\
\hline $\mathrm{CP}$ & $85.39^{\mathrm{b}}$ & $84.61^{b}$ & $76.95^{\mathrm{ab}}$ & $73.89^{\mathrm{a}}$ & 1.397 & $<0.01^{* *}$ & 0.584 \\
\hline $\mathrm{EE}$ & 72.24 & 68.49 & 64.71 & 69.02 & 1.266 & 0.229 & 0.115 \\
\hline $\mathrm{CF}$ & 59.99 & 65.88 & 65.59 & 67.50 & 1.462 & 0.105 & 0.496 \\
\hline NFE & $88.21^{\mathrm{b}}$ & $84.12^{\mathrm{ab}}$ & $84.06^{\mathrm{ab}}$ & $78.26^{\mathrm{a}}$ & 1.166 & $<0.01^{* *}$ & 0.607 \\
\hline \multicolumn{8}{|l|}{ Economics } \\
\hline Av. total feed intake (kg) & 176.52 & 177.76 & 171.54 & 179.97 & 1.749 & 0.793 & 0.320 \\
\hline Av. total feed cost (Rs) & $5052.80^{a}$ & $4911.40^{\mathrm{ab}}$ & $4561.80^{\mathrm{b}}$ & $4588.10^{\mathrm{b}}$ & 63.55 & $<0.01^{* *}$ & 0.402 \\
\hline Av. total weight gain $(\mathrm{kg})$ & 40.04 & 40.13 & 41.44 & 40.16 & 1.000 & 0.860 & 0.754 \\
\hline Cost of feed / kg weight gain (Rs) & 118.28 & 117.66 & 116.17 & 108.69 & 2.799 & 0.247 & 0.559 \\
\hline
\end{tabular}

Note: DM - dry matter; OM - organic matter; CP - crude protein; EE - ether extract; CF - crude fibre; NFE - nitrogen free extract; Av. - average; a,b means with different superscripts in the same row differ significantly (** $p<0.01)$; SEM - standard error of mean.

\section{DISCUSSION}

\section{Chemical Composition of POS and Experimental Feeds}

The $\mathrm{CP}$ values obtained in the present study were similar to the observation made by Habib et al. (1997), Wong and Wan Zahari (1992) and Kekiepeu (2017). They found the CP content in POS was $12.75 \%, 12.50 \%$ and $14.30 \%$ respectively. However, the $\mathrm{CP}$ value in the present study contradicts with Devendra (1977); Bobadoye et al.
(2008) and Faradonbeh et al. (2011) who reported lower values in their works, which could be due to the processing methods of oil extraction. The ether extract content of POS in our study was similar to the values reported by Habib et al. (1997) and Kekiepeu (2017) but lesser than the values observed by Devendra (1977); Bobadoye et al. (2008) and Faradonbeh et al. (2011). The CF value was similar to the findings of Wong and Wan Zahari (1992) but higher than the values reported by Bobadoye et al. (2008) and Faradonbeh et al. (2011). The NFE value was similar to the values reported by Habib et al. 
(1997); et al. (2008) and Faradonbeh et al. (2011). In the present study, the total ash in POS was similar to the findings of Wong and Wan Zahari (1992) and Kekiepeu (2017) but lesser than the observation made by Earp and Newal (1976); Abu et al. (1984) and Wan Zahari and Alimon (2003). The AIA content was found to be high $\left(72.80 \mathrm{~g} \mathrm{~kg}^{-1} \mathrm{DM}\right)$ in POS. POS is the decanted form of POME, which consists of liquids, dirt, residual oil and suspended solids, mainly cellulosic material from the mesocarp of the fruits. Hence, the high ash content of the sludge may be due to the contamination by the soil or suspended solids from the mesocarp. The NDF and ADF values in this study were lower than the values reported by Bamikole and Babayemi (2008). The wide variability in the chemical composition of POS is due to the oil palm varieties (Bamikole and Ikatua, 2009) and the method of oil extraction (Seephueak et al., 2011).

The major long chain fatty acids in POS were myristic, palmitic, stearic, oleic and linoleic acid. The palmitic acid (g per $100 \mathrm{~g}$ fatty acid) content was highest in POS (53.38 g). The SFA was found to be higher in comparison to MUFA and PUFA in POS. In this study, palmitic, oleic and linoleic acid. were found to be higher than the findings of Habib et al. (1997) where the corresponding values of $22.45 \%, 15.54 \%$ and $9.53 \%$ was reported in raw POME. Myristic and stearic was higher in our study compared to their observation. Abubakr et al. (2015) reported that palmitic, stearic, oleic and linoleic acid in palm oil decanter cake were found to be $49.64 \%$, $3.76 \%, 35.40 \%$ and $7.34 \%$, respectively, where stearic acid level was comparable but oleic acid level was lower than the present study. Moreover, C18:0 (8.06 $\mathrm{g})$ and $\mathrm{C} 18: 2 \mathrm{cis}(10.13 \mathrm{~g})$ content was higher than the values observed in our study.

As shown in Table 2, the experimental pigs were fed in two Phases (I and II). The Phase I (20-50 kg) and Phase II (50-80 kg) diets for all the experimental groups was iso-nitrogenous and iso-caloric. However, the EE, CF and TA content increased with increasing level of POS in the diets of both the phases. This may be attributed to higher CF and ash content of POS and the incorporation of palm oil in the concentrate diets. Above all, the concentrate diets in Phase I and II feeding adhered with the specification of NRC 1998.

\section{Feed and Nutrient Intake}

There was no significant difference in DMI (g $\mathrm{hr}^{-1}$ day $^{-1}$ ) in the whole experimental period. Thus, the performance of the pigs was not adversely affected by inclusion of graded levels of POS as a replacement of maize in both the phase of feeding (Phase I and II). However, DMI ( $\mathrm{g} \mathrm{hr}^{-1} \mathrm{day}^{-1}$ ) partially tended to increase in the treatment groups except Group-3 which could be due to reduced dustiness and enhanced palatability of POS. The intake was optimum in all the treatment groups which indicated that the experimental pigs had adapted to the POS based diets. Our finding were similar to the findings of Abu et al. (1984) who observed no changes in the feed intake of pigs when oil palm slurry was fed as a partial replacement for maize. Similarly, Fanimo and Fashina-Bombata (1998) also reported similar feed intake in weaner pigs fed iso-nitrogenous diets containing various levels $(0 \%, 10 \%, 15 \%, 20 \%$ and $25 \%$ ) of POS.

\section{Body Weight Changes and Feed Conversion Ratio}

The replacement of maize with POS had no effect on the body weight gain, ADG and FCR in both the feeding phases. Regardless of the inclusion levels of POS, the performance was not impaired and thus further establishes its use in concentrate diet as an energy source for growingfinishing pigs. This is due to enhanced palatability and the addition of crude palm oil had reduced the dustiness of the feed in the study. The weight gain and feed efficiency in the present observation is in concurrence with the finding of Abu et al. (1984) and Enemona (2008) who reported non-significant difference in pigs when maize was replaced up to $30 \%$ by POS in the basal diets. Similarly, feeding of palm kernel cake, another by-product of oil palm to pigs (up to $59.05 \mathrm{~kg}$ ) at the level of $50 \%$ and $100 \%$ to replace maize did not influence the performance of the pigs (Adesehinwa, 2007).

\section{Blood Profiles}

The values were within the normal range for pigs described by Latimer et al. (2003) for $\mathrm{MCH}$ and Radostits et al. (2000) for MCHC and monocyte. There was no anaemic and immunosuppressive pigs in the herd during the whole experimental period and the reason for this may be due to individual variation within the groups. In contrast, Kekiepeu (2017) reported no variation $(p>0.05)$ in the $\mathrm{MCH}, \mathrm{MCHC}$ and monocyte concentrations in poultry when maize was replaced at various levels $(0 \%, 5 \%, 10 \%$ and $15 \%)$ with POS in the concentrate diets.

All the serum biochemical parameters were within the physiological range for pigs. The present observation is in agreement with the findings of Kekiepeu (2017) who reported non-significant ( $p>0.05)$ difference in serum metabolites (SGPT, SGOT, total protein and glucose) of broiler poultry fed POS at 5\%,10\% and 15\% levels replacing maize. On the contrary, Onibi et al. (2011) observed that the serum cholesterol levels increased significantly $(p<0.05)$ in chickens with increasing levels of POS in diets and further suggested that POS inclusion in the diets could lead to hypercholesterolmanic tendency in birds. 


\section{Apparent Digestibility of Nutrients}

The digestibility indices decreased with the increase of POS level in all the test diets and the apparent digestibility of components were higher in Group-1 and lower in Group-4. The reason could be due to high content of ash and CF in the test ingredient. This is further supported by the fact that presence of CF affect the CP digestibility (Sauer et al., 1980) and the excretion of fecal nitrogen compared to urinary nitrogen is increased when the total dietary fibre level is increased (Bindelle et al., 2009). However, several reports suggest that when the source of fibre does not contribute significant amounts of protein to the diet, then an increase in the level of fibre to some extent does not affect protein digestibility significantly (Gouwens, 1966; Friend, 1970; Eggum, 1973; Kennelly and Aherne, 1980). Our findings are in conformity with Fanimo and Fashina-Bombata (1998) who observed decreased CP digestibility in the diet of weaner pigs fed POS based concentrate diets. The digestibility coefficient of $\mathrm{EE}$ and $\mathrm{CF}$ was found to be non-significant ( $p>0.05)$ among the treatments. The higher digestibility CF could be due to high intact or extracted fat (Kil et al., 2010) for EE and more of soluble dietary fibre for CF in the diets. In our study, fibre rich POS was used with high energy feed ingredients i.e., crude palm oil to maintain the DE content in the treatment diets. Dietary fat might have increased the retention time of digesta, which prolonged the digesta fermentation by the gut microbiota (Wilfart et al., 2007). The finding is in agreement with Adesehinwa (2007) who reported that there was no significant difference $(p>0.05)$ in apparent digestibility of crude fibre in growing pigs at $50 \%$ and $100 \%$ replacement of maize with palm kernel cake.

\section{Economics}

The feed cost was higher in Group-1 and lower in Group-4. This shows that it was far more economical to use POS to replace maize in the pig diets as it cost little when compared to maize. Although, there was no significant $(p>0.05)$ difference in the feed cost per $\mathrm{kg}$ weight gain, the least cost per $\mathrm{kg}$ weight gain was observed for pigs on $30 \%$ replacement of maize by POS. Economically, incorporation of POS up to $30 \%$ level replacing maize was found to be cost effective and can be used as a feed for growing-finishing pigs in those areas where POS is readily available and affordable. Similar finding was observed by Hertrampt (1988) and Enamona (2008) in pigs. Likewise, Adesehinwa (2007) reported that feeding of palm kernel cake (PKC), which is also a by-product of oil palm revealed significantly $(p<0.05)$ lower feed cost as the level of PKC inclusion increase. There was no significant $(p>0.05)$ difference in the feed cost per live weight gain between the various treatment groups. In contrast, Ezewke et al. (2011) reported that feed cost per $\mathrm{kg}$ gain was lower and better for pigs fed diets containing 15\% and 30\% POS diets in growing pigs.

\section{CONCLUSION}

On the basis of the above results, it can be concluded that feeding of POS as partial replacement of maize up to $30 \%$ in the basal diet had no adverse affect on the performance and the haematobiochemical parameters of the growing finishing pigs. Hence, POS can be used as a substitute for costly feed ingredients like maize in the diet of pigs where it is easily available to minimise the cost of production. However, research can be carried out on supplementation of enzymes in POS based diets to further improve the digestibility of nutrients.

\section{ACKNOWLEDGEMENT}

The authors thank the Central Agricultural University, Imphal and the Dean, College of Veterinary Sciences and Animal Husbandry, Selesih, Aizawl, Mizoram for providing necessary facilities to carry out the research.

\section{REFERENCES}

Abubakr, A; Alimon, A R; Yaakub, H; Abdullah, $\mathrm{N}$ and Ivan, $\mathrm{M}$ (2015). Effect of feeding palm oil by-products based diets on muscle fatty acid composition in goats. PLOS ONE, 10(3): e0119756. DOI:10.1371/journal.pone.0119756.

Abu, A A; Okai, D B and Tuah, A K (1984). Oil palm slurry (OPS) as a partial replacement for maize in the diets of growing-finishing pigs. Nut. Rep. Int., 30(1): 121-127.

Adesehinwa, A O K (2007). Utilization of palm kernel cake as a replacement for maize in diets of growing pigs: Effects on performance, serum metabolites, nutrient digestibility and cost of feed conversion. Bulg. J. Agric. Sci., 13: 593-600.

AOAC (1995). Official Methods of Analysis. 16 $6^{\text {th }}$ edition. Association of Official Analytical Chemists, Washington, DC.

Bobadoye, A O; Onibi, G E; Fajemisin, A N; Olasupo, $\mathrm{O} O$ and Bobadoye, B O (2008). Replacing maize with palm oil sludge in broiler chicken diets: Effect on Carcass characteristics, organ weight and muscle development. Int. J. Sustain. Crop Prod., 3(6): 1-5. 
Bamikole, M A and Babayemi, O J (2008). Chemical composition and in sacco dry matter degradability of residue and by-products of palm fruit processing in the rumen of steers. Anim. Sci. J., 79: 314-321.

Bamikole, M A and Ikatua, U J (2009). Variety diversity effect on the chemical composition and dry matter degradation characteristics of residue and by-products of oil palm fruits. Anim. Sci. J., 80: 239-249.

Bindelle, J; Buldgen, A; Delacollette, M; Wavreille, J; Agneessens, R; Destain, J P and Leterme, P (2009). Influence of source and concentrations of dietary fiber on in vivo nitrogen excretion pathways in pigs as reflected by in vitro fermentation and nitrogen incorporation by fecal bacteria. J. Anim. Sci., 87: 583593.

Devendra, C (1977). Utilization of feeding stuffs from the oil palm. Proc. Symp. Feedingstuffs for Livestock in South East Asia. p. 116-131.

Earp, D A and Newal, W (1976). International Development in Palm Oil. The Incorporated Society of Planters. Kuala Lumpur, Malaysia. Edmard Arnold Pub, London. p. 131-135.

Eggum, B O (1973). A study of certain factors influencing protein utilization in rats and pigs. Ph.D thesis, National Institute of Animal Science, Copenhagen, Denmark.

Enemona, J (2008). Growth performance of weaner pigs fed processed and unprocessed palm oil sludge. M.Sc thesis, University of Nigeria, Nsukka, Nigeria.

Ezekwe, A G; Machebe, N S and Enemona, J (2011). Performance and cost benefit of substituting palm oil sludge (POS) for maize in diets of grower pigs. Int. J. Sci. Nat., 2(2): 210-214.

Fanimo, A O and Fashina-Bombata, H A (1998). The response of weaner pigs to diets containing palm oil slurry. Anim. Feed Sci. Technol., 71: 191-195.

Faradonbeh, P O; Bagheri, $\mathrm{H}$ and Soleimani, $\mathrm{R}$ (2011). Efficacy of palm oil on commercial chicks: Performance traits. Res. Opin. Anim. Vet. Sci., 1(12): 766-769.

Goering, H K and Van Soest, P J (1970). Forage fiber analysis. USDA Agriculture Handbook No. 379. USDA-ARS, Washington, DC, USA.

Gouwens, D W (1966). Influence of Dietary Protein and Fiber on Fecal Amino Acid Excretion of Swine. M.Sc thesis, University of Illinois, Urbana.
Friend, D W (1970). Comparison of some milling products of barley and rye when fed in diets to rats. Can. J. Anim. Sci., 50: 345-348.

Habib, M A B; Yusoff, F M; Phang, S M; Ang, K J and Mohamed, S (1997). Nutritional values of chironomid larvae grown in palm oil mill effluent and algal culture. Aquaculture, 158: 95-105.

Hertrampt, J W (1988). Unconventional feedstuffs for livestock. Die Mühle - Mischfuttertechnik, 125(9): 108-109.

Kekiepeu, N (2017). Study on the performance of broiler chicken fed on partially maize replaced diet with palm oil (Elaeis guineensis) sludge. Thesis, Department of Animal Nutrition, College of Veterinary Sciences and Animal Husbandry, Central Agricultural University, Aizawl, Mizoram, India.

Kennelly, J J and Aherne, F X (1980). The effect of fiber addition to diets formulated to contain different levels of energy and protein on growth and carcass quality of swine. Can. J. Anim. Sci., 60: 385-393.

Kil, D Y; Sauber, T E; Jones, D B and Stein, H H (2010). Effect of the form of dietary fat and the concentration of dietary neutral detergent fiber on ileal and total tract endogenous losses and apparent and true digestibility of fat by growing pigs. J. Anim. Sci., 88: 2959-2967.

Latimer, K S; Mahaffey, E A and Prasse, K W (2003). Duncan and Prasse's Veterinary Laboratory Medicine: Clinical Pathology. Fourth edition. WileyBlackwell.

Mandal, G P; Roy, A and Patra, A K (2014). Effects of feeding plant additives rich in saponins and essential oils on the performance: Carcass traits and conjugated linoleic acid concentrations in muscle and adipose tissues of Black Bengal goats. Anim. Feed Sci. Technol., 197: 76-84.

MOFPI (2017). Mizoram State Report 2017. Ministry of Food Process Industry (MOFPI). New Delhi, India.

Noblet, J and Perez, J M (1993). Prediction of digestibility of nutrients and energy values of pig diets from chemical analysis. J. Anim. Sci., 71: 33893398.

National Research Council (1998). Nutrient Requirement of Swine. $10^{\text {th }}$ edition. National Research Council, National Academy Press, Washington DC. 
O'Fallon, J V; Busboom, J R; Nelson, M L and Gaskins, C T (2007). A direct method for fatty acid methyl ester synthesis: Application to wet meat tissues, oils and feedstuffs. J. Anim. Sci., 85: 15111521.

Onibi, G E; Bobadoye, A O and Folorunso, O R (2011). Haematological indices, serum cholesterol and meat quality of broiler chickens fed diets with palm oil sludge substituting maize. Agric. Biol. J. North America, 2(3): 552-558.

Perez, R (1997). Feeding pigs in the tropics. FAO Animal Production and Health Paper 132. Food and Agriculture Organization of United Nations (FAO). Rome, Italy.

Radostits, O M; Gay, C C; Blood, D C and Hinchcliff, K W (2000). Veterinary Medicine. $9^{\text {th }}$ edition. W B Saunders, London. p. 1819-1822.

SAS (2003). Statistical Analytical System. SAS Institute Inc Cary. North Carolina, USA.

Sauer, W C; Just, A; Jergensen, H H; Fekadu, M and Eggum, B O (1980). The influence of diet composition on the apparent digestibility of crude protein and amino acids of the terminal ileum and overall in pigs. Acta Agric. Scand., 30: 449-459.

Seephueak, W; Ngampongsai, W and Chanjula, P (2011). Effects of palm oil sludge in concentrate on nutrient utilization and rumen ecology of Thai native cattle fed with hay. Songklanakarin J. Sci. Technol., 33(3): 271-280.
Talapatra, S K; Ray, S C and Sen, K C (1940). Estimation of phosphorous, chlorine, calcium, magnesium, sodium and potassium in food-stuffs. J. Vet. Sci., Anim. Husb., 10: 243-246.

Wan Zahari, M and Alimon, A (2003). Use of palm kernel cake and oil palm by-products in compound feed use of palm kernel cake and oil palm byproducts in compound feed. Palm Oil Developments, 40: 5-9.

Webb, B H; Hutagalung, R L and Chean, S $\mathrm{T}$ (1977). Palm oil mill waste as animal feed. Processing and Utilization (Earp, D A and Nawal, W eds.). International Development in Palm Oil. Incorporated Society of Planters. Kuala Lumpur. p. 125-145.

Wilfart, A; Montagne, L; Simmins, H; Noblet, J and van Milgen, J (2007). Digesta transit in different segments of the gastrointestinal tract of pigs as affected by insoluble fiber supplied by wheat bran. Br. J. Nutr., 98: 54-62.

Wong, H K and Wan Zahari, M (1992). Characterization of oil palm by-products as feed for ruminants. Proc. of the $15^{\text {th }}$ Annual Conference of Malaysian Society of Animal Production. Terengganu, Malaysia. p. 58-61.

Vroom, S J (1978). The effects of different levels of oil palm slurry (OPS) on the growth rate and performance of weaner pigs. Unpublished. Dissertation, University of Science and Technology, Department of Animal Science, Kumasi, Ghana. 